Collection SFN 10 (2010) 631-649

(C) Owned by the authors, published by EDP Sciences, 2010

DOI: $10.1051 / \mathrm{sfn} / 2010017$

\title{
Transition isolant-métal induite par dopage dans les manganites, sondée par les ondes de spin
}

\author{
M. Hennion
}

\author{
Laboratoire Léon Brillouin, CE-Saclay, 91190 Gif-sur-Yvette, France
}

\begin{abstract}
Résumé. Nous présentons une étude des excitations magnétiques dans des composés de $\mathrm{La}_{1-x} \mathrm{Sr}_{x} \mathrm{Mn} \mathrm{O}_{3}$ et $L a_{1-x} \mathrm{Ca}_{x} \mathrm{MnO}_{3}$ où $x$ varie de manière à couvrir une large partie des diagrammes de phases. Dans l'état antiferromagnétique, $0.1<x<0.1$, deux branches d'ondes de spin sont observées indiquant l'existence de deux dynamiques de spins dans des domaines d'énergie distincts, connectés à $q=0.25$, tandis que la diffusion aux petits angles révèle des amas bidimensionnels de taille $\xi=16 \AA$, indiquant une ségrégation de charges. Au-delà, lorsque les composés deviennent ferromagnétiques, la courbe d'ondes de spin présente de fortes anomalies. Trois régions distinctes apparaissent selon $x$ et $\mathrm{T}$ : quasi-métallique pour $\mathrm{x} \approx 1 / 8$ et $T o o^{\prime}<T<T c$, isolante pour $x \approx 1 / 8$ et $\left.T<T o o^{\prime}\right)$ et métallique pour $x(S r)>0.15$ ou $x(C a)>0.22$. Dans ces régions, les spectres d'ondes consiste en une dispersion quadratique à petit vecteur d'onde, et des niveaux à l'approche du bord de zone, caractéristiques de "défauts magnétiques" locaux. L'ensemble de ces anomalies pourrait s'expliquer par la mobilité de la paire de spins de Zener assistée par les phonons du réseau.
\end{abstract}

\section{INTRODUCTION}

Après les premiers travaux de Wollan et Koelher [1], la découverte de phénomènes de magnéto-transport exceptionnels a entrainé un regain d'activité pour l'étude des manganites. Dans les composés à dopage en trous de l'ordre de $30 \%$, la résistivité peut changer de plusieurs ordres de grandeur sous l'effet d'un champ magnétique [2,3]. L'intérêt et l'originalité de ces composés viennent en fait de ce que la transition paramagnétique-ferromagnétique est associée à une transition isolant-métal, c'est à dire à un profond changement de la structure électronique. Une deuxième motivation provient de la richesse de leur diagrammes de phase. Une grande variété de structures apparaît avec des phénomènes inhabituels d'ordre pour les spins et les charges, en liaison avec un ordre des orbitales, qui constituent les degrés de liberté de ces systèmes. Une autre motivation vient de l'apparition d'une physique nouvelle, dévoilée à la fois par les expériences et les théories: la ségrégation de charge à l'échelle nanoscopique [4]. En l'absence de lien établi entre toutes les observations, la véritable origine des effets exceptionnels de magnéto-transport reste encore largement incomprise.

De manière surprenante, il y a eu peu d'études de neutrons sur les phases "précurseures" de cette phase métallique, en fonction de la concentration. C'est cette approche que nous décrivons ici à partir du composé pur $\mathrm{LaMnO}_{3}$, antiferromagnétique isolant, par dopage progressif en trous, obtenu par substitution de $\mathrm{La}^{3+}$ par $\mathrm{Ca}^{2+}$ ou $\mathrm{Sr}^{2+}$ jusqu'à la phase ferromagnétique métallique.

Comme l'indique les diagrammes de phases de $\mathrm{La}(\mathrm{Ca}, \mathrm{Sr}) \mathrm{MnO}_{3}$ de la figure 1, 5 états magnétiques différents sont rencontrés, que nous décrirons successivement :

- L'état du composé pur $\mathrm{LaMnO}_{3}$, antiferromagnétique à $\mathrm{T}_{N}=140 \mathrm{~K}$ et isolant

- L'état canté antiferromagnétique, isolant, pour $0>x>0.1$

This is an Open Access article distributed under the terms of the Creative Commons Attribution-Noncommercial License 3.0, which permits unrestricted use, distribution, and reproduction in any noncommercial medium, provided the original work is properly cited. 


\section{Diagrammes de phase: 5 états « magnétiques»}
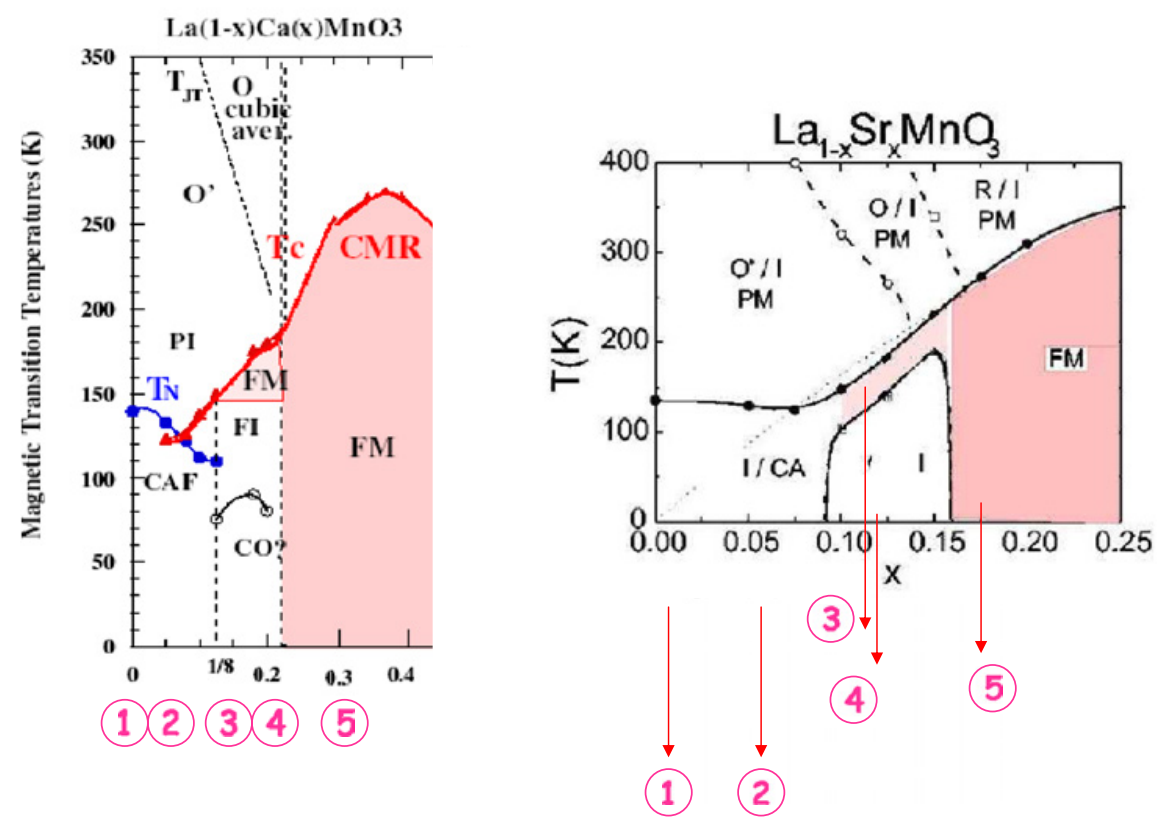

Figure 1. Diagrammes de phases de $L a_{1-x} \mathrm{Ca}-x \mathrm{MnO}_{3}$, à gauche et $L a_{1-x} \mathrm{Sr}_{x} \mathrm{Mn} \mathrm{O}_{3}$ à droite. La couleur blanche indique un caractère isolant et rose un caractère métallique. 5 états magnétiques distincts apparaissent, similaires dans les 2 cas, décrits dans le texte. Les lignes $\mathrm{O}, \mathrm{O}^{\prime}, \mathrm{R}$ indiquent des transitions structurales de type orthorhombique $(\mathrm{O})$ et rhomboédrique $(\mathrm{R})$.

- L'état ferromagnétique quasi-métallique qui apparait à $x \approx 1 / 8$ dans un domaine limité de température $\mathrm{T}_{C}<T<T_{O O^{\prime}}\left(T_{O O^{\prime}}\right.$ caractérise une anomalie de résistivité associée à une transition structurale)

- L'état ferromagnétique "isolant" pour $x \approx 1 / 8$ et $T<T_{O O}$

- L'état ferromagnétique métallique.

\section{CARACTÉRISTIQUES GÉNÉRALES DES MANGANITES}

\subsection{Diagrammes de phase partiels de $\mathrm{La}_{1-\mathrm{x}} \mathrm{Sr}_{\mathrm{x}} \mathrm{MnO}_{3}$ et $\mathrm{La}_{1-\mathrm{x}} \mathrm{Ca}_{\mathrm{x}} \mathrm{MnO}_{3}$}

Nous avons choisi de comparer deux systèmes a priori semblables mais pour lesquels les effets de magnétotransport sont quantitativement différents, les composés substitués au Sr et au Ca. Dans la figure 1 sont représentés les diagrammes de phases de $L a_{1-x} C a_{x} M n O_{3}$ et $L a_{1-x} S r_{x} M n O_{3}$, depuis le composé pur $\mathrm{La} \mathrm{MnO}_{3}$, antiferromagnétique jusqu'à l'état métallique ferromagnétique. Les différentes couleurs, de blanche pour isolant, à rose clair pour "quasi-métallique", puis enfin rose foncé pour un état véritablement métallique, symbolisent les propriétés de transport. Cinq états magnétiques, très similaires dans les deux types de composés, sont observés, avec des valeurs légèrement différentes pour les températures de transition magnétiques ou structurales ainsi que pour les concentrations "critiques". Ces différences résultent de la différence de taille entre les atomes $\mathrm{Ca}$ et $\mathrm{Sr}$. Dans ces diagrammes, les notations $O^{\prime}$ ou $O$ indiquent une structure orthorhombique, et $R$ une structure rhomboédrique. Toutes ces structures sont dérivées de la structure cubique pérovskite de base. La distorsion orthorhombique 


\section{Résistivité et magnétorésistance}

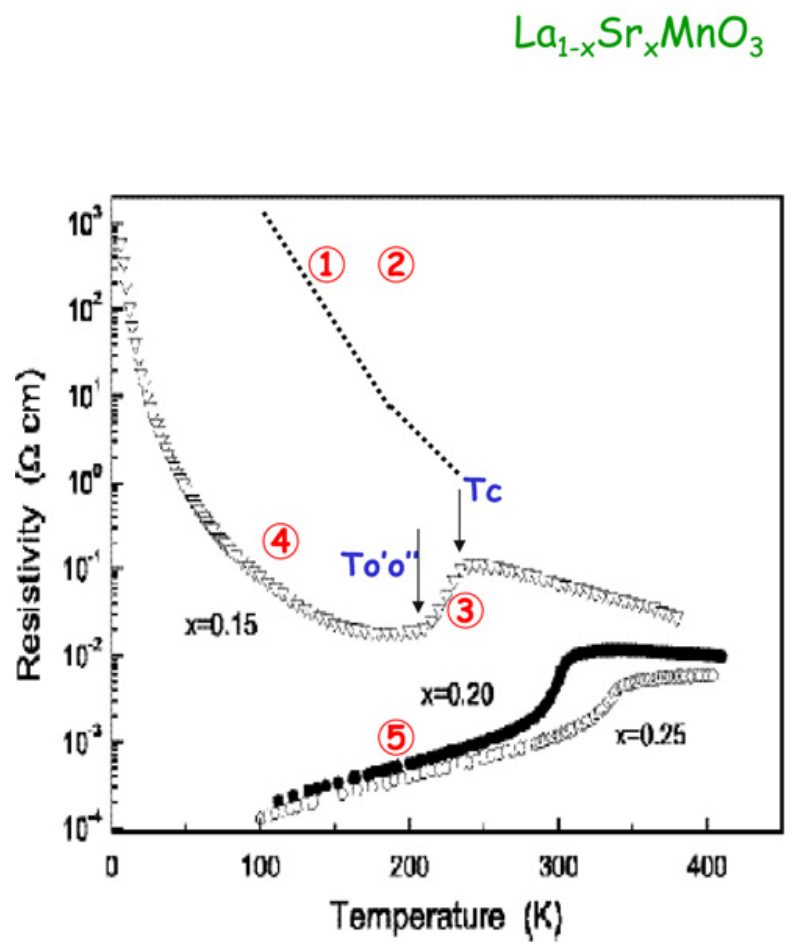

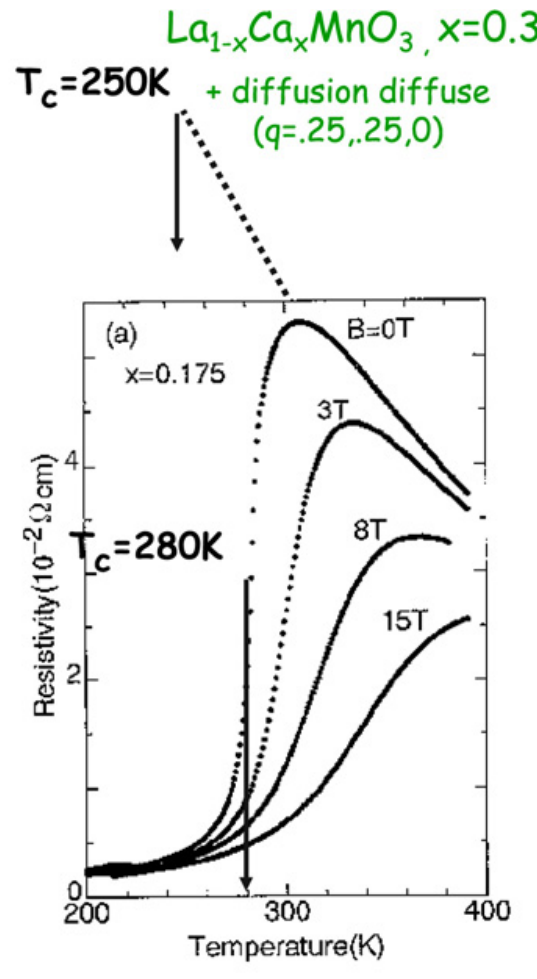

Figure 2. A gauche évolution de la résistivité avec le dopage, permettant de définir les zones du diagramme de phases de la figure 1. A droite variation de la résistivité avec le champ magnétique (magnétoresistance) du composé $L a_{1-x} S r_{x} M n O_{3} \times(S r)=0.175$. En pointillé, pour comparaison, courbe schématique de la résistivité du composé $L a_{1-x} \mathrm{Ca}_{x} \mathrm{MnO}_{3}, \mathrm{x}(\mathrm{Ca})=0.3$.

est maximale pour le composé pur $\mathrm{LaMnO}_{3}$, en liaison avec la différence de taille entre La et $\mathrm{Mn}$, et se réduit progressivement par l'effet de substitution. Les lignes de transition structurale ayant peu d'incidence sur la physique qui nous intéresse ici, elles ne sont indiquées que dans la région paramagnétique et non dans les régions ferromagnétiques à plus basse température, où elles se prolongent.

\subsection{Bases théoriques}

Les données théoriques de base permettant de comprendre les propriétés moyennes des manganites sont reportées dans la figure 3, qui détaille l'état électronique de l'ion Jahn-Teller $\mathrm{Mn}^{3+}$. Dans une approximation ionique, $L a$ est trivalent (3+) et $O$ divalent (2+), l'atome de Mn est alors trivalent, ce qui signifie qu'il existe 4 électrons de type "d". Le niveau $d$ correspond à 5 états électroniques (ou 10 si l'on inclut l'état de spin). Lorsque l'ion Mn est isolé, ces états électroniques ont la même énergie. Sous l'effet du champ cristallin créé par les octaèdres d'oxygène de la structure pérovskite (ronds bleus de la figure -3 , au centre), les niveaux d'énergie de la bande $d$ se subdivisent en 2 , avec un niveau profond, $t_{g}$, correspondant aux 3 orbitales de type $x y$, et un niveau extérieur, $e_{g}$, comprenant 2 types d'orbitale: $\left(x^{2}-y^{2}\right)$, avec des maxima pointant le long des diagonales du plan, et $\left(3 z^{2}-r^{2}\right)$, de forme allongée selon les axes du petit cube (représentées dans la figure 3, au centre). 

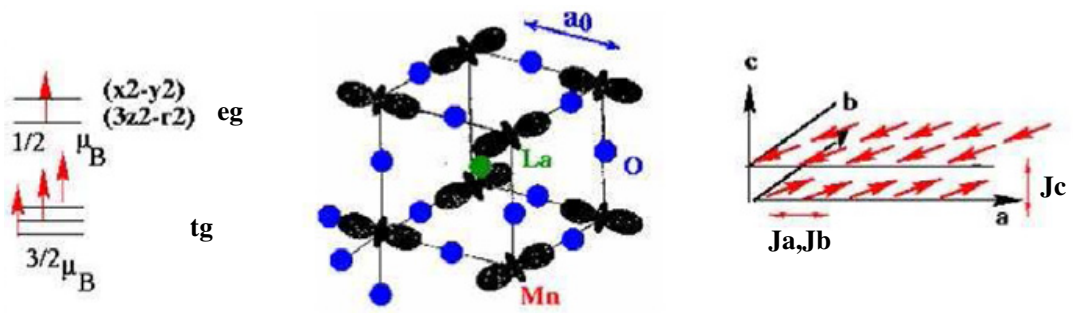

Figure 3. $\mathrm{LaMnO}_{3}$ : A gauche, état electronique de $\mathrm{Mn}$, au centre, ordre orbital, à droite, structure magnétique.

a)

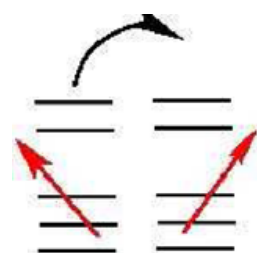

b)

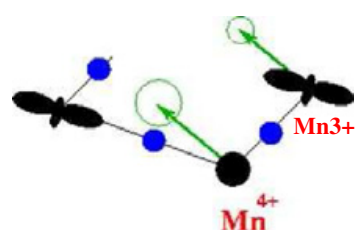

c)

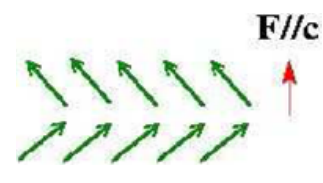

Figure 4. Représentation schématique de l'évolution avec le dopage : A gauche: saut de l'electron, au centre: ordre orbital, a droite: ordre magnétique.

$\mathrm{Si}$ on applique les règles dites de Goodenough-Kanamori où deux orbitales $\left(3 z^{2}-r^{2}\right)$ orientées à 90 degrés créent un couplage ferromagnétique et deux orbitales parallèles créent un couplage antiferromagnétique, on peut expliquer l'origine de la structure antiferromagnétique qui apparait à $\mathrm{T}_{N}=140 \mathrm{~K}$ (cf dessin à droite). Cet ordre antiferromagnétique dit de type "A", consiste en plans ferromagnétiques (a, b) empilés antiferromagnétiquement, le long de l'axe c. L'étude des excitations magnétiques dans $\mathrm{LaMnO}_{3}$ nous permet de dire que le couplage est équivalent suivant $\mathbf{a}$ et $\mathbf{b}$. On définit donc $J_{a}=J_{b}$ suivant a ou b et $J_{c}$ suivant $\mathbf{c}$.

La substitution de $\mathrm{La}^{3+}$ par $\mathrm{Ca}^{2+}$ ou $\mathrm{Sr}^{2+}$, correspond à un dopage en trous, et donc à l'apparition d'ions $\mathrm{Mn}^{4+}$ sans orbitale extérieure $e_{g}$. Ces electrons (ou trous) vont sauter d'un atome de Mn sur l'atome voisin. L 'effet du dopage correspond à l'introduction d'un nouveau couplage dit "doubleéchange" de nature ferromagnétique [5,6]. A cause de la règle de Hund, selon lequel le saut de l'électron (ou du trou) est favorisé par l'alignement des spins du coeur, les trous se déplacent plus facilement dans les plans ferromagnétiques que selon l'axe c. Les sauts de charge vont cependant modifier la structure magnétique moyenne, et le système évolue d'une structure ferromagnétique en couches (plans a, $\mathbf{b}$ ), $A F$ selon c, vers une structure plus isotrope, avec l'apparition d'une composante ferromagnétique selon la direction c. L'état est dit “canté” (Fig 4-c).

\section{EXPÉRIENCES}

\subsection{Mesure}

Les mesures d'ondes de spin se font presque obligatoirement sur des monocristaux. Grâce à l'état monocristallin, on peut définir un réseau réciproque, et par l'orientation du monocristal, choisir le plan du réseau réciproque qui nous intéresse pour les mesures de diffusion inélastiques des neutrons. Les valeurs $\omega(\mathrm{q})(Q=q+\tau)$ des excitations magnétiques à déterminer, sont identiques dans toutes les zones de Brillouin construites autour des pics de Bragg $\tau$. Seule l'intensité change. 


\section{(1) ondes de spin dans $\mathrm{LaMnO}_{3} \quad$ F. Moussa et al Phys. Rev. B 54,15149 1996}

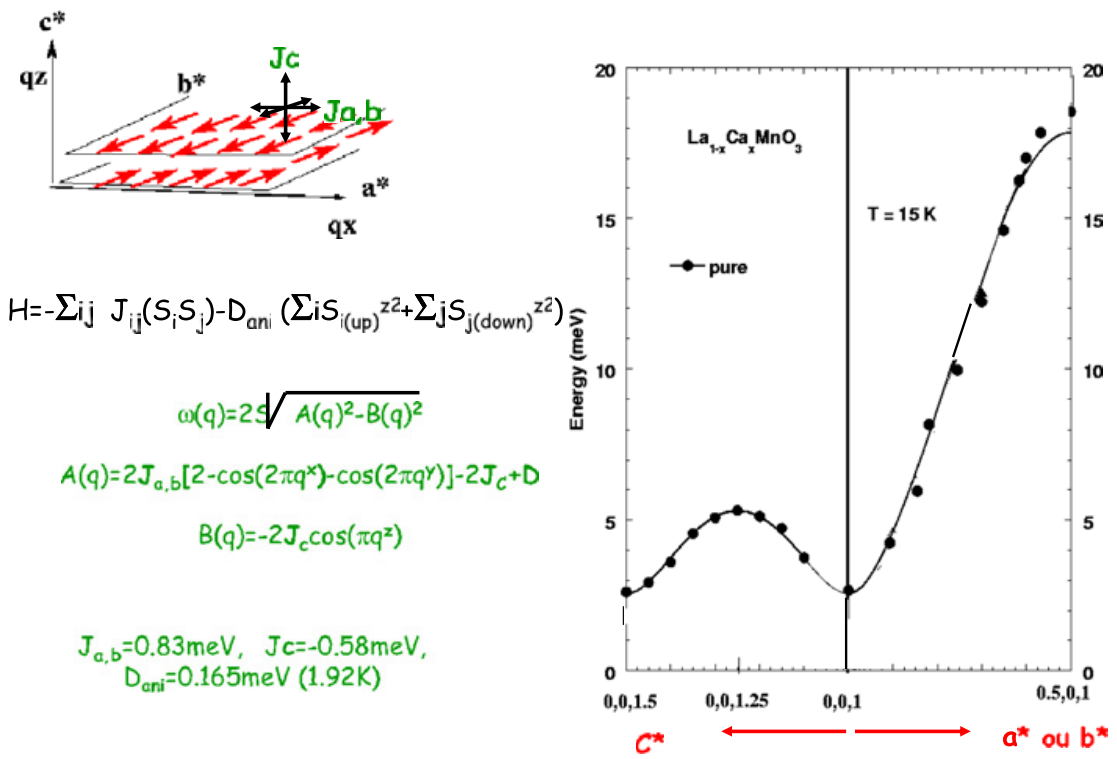

Figure 5. Dispersion $\omega$ (q) du composé pur selon a ou b et selon c.

\section{2 État 1 : composé pur $\mathrm{LaMnO}_{3}$}

Sur la figure 5, on a représenté :

- à droite, la mesure de la courbe de dispersion selon l'axe cet selon l'axe a ou b (les 2 directions étant équivalentes) [7]. Pour simplifier, on a considéré les vecteurs d'onde du plan (a, b) dans l'indexation de la structure pseudo-cubique et non orthorhombique (qui correspond en fait à la structure réelle). La maille magnétique considérée est donc un carré de coté $a$ dans le plan, et de longueur $2 a$ le long de c. Trois observations sont faites immédiatement:

- La période du couplage magnétique dans la direction $\mathbf{c}$ est deux fois celle des directions a ou $\mathbf{b}$. Cela indique un couplage antiferromagnétique selon $\mathbf{c}$ et ferromagnétique selon $\mathbf{a}$ ou $\mathbf{b}$, en accord avec la structure magnétique.

- La comparaison des domaines d'énergie des 2 directions indique un couplage plus faible selon c que selon $\mathbf{a}$ ou $\mathbf{b}$.

- La forte énergie à l'origine $(q=0)$ indique une forte anisotropie magnétocristalline. En fait, on peut donner une interprétation plus quantitative en utilisant un Hamiltonien de Heisenberg.

- On a représenté, Figure 5 (gauche), deux plans de la structure magnétique du composé pur $\mathrm{LaMnO}_{3}$, avec les constantes de couplage premiers voisins $\mathbf{J}_{a}$, $\mathbf{J}_{b}$ dans le plan (a, b) et $\mathbf{J}_{c}$ selon $\mathbf{c}$. Un atome de Mn est donc lié dans le plan à 4 atomes de $\mathrm{Mn}$ avec $\mathrm{J}_{a}$ et, selon $\mathbf{c}$, à 2 atomes avec $\mathrm{J}_{c}$. Dans la structure magnétique de $\mathrm{LaMnO}_{3}$, la direction du spin est attachée à une direction particulière du plan (pour faciliter le dessin on a mis l'axe du spin selon une direction du cube, alors qu'en réalité, le spin est selon la direction $(\mathbf{a}+\mathbf{b})$ du plan). Cette anisotropie, uniaxiale, est définie par $D_{a n i}$. La diagonalisation de l'Hamiltonien, qui permet d'obtenir les modes propres, se fait analytiquement. La courbe de dispersion $\omega(q)$, fait intervenir deux fonctions $A(q)$ et $B(q)$, dont l'expression analytique est donnée dans la figure 5. Les valeurs absolues du couplage ferromagnétique et antiferromagnétique sont assez différentes $(0.83 \mathrm{meV}$ et $-0.58 \mathrm{meV})$ [7]. On peut remarquer, par les expressions analytiques, que 
l'énergie à $q=0$ n'est pas directement la valeur de l'anisotropie $D_{a n i}$, mais une fonction de $D_{a n i}$ et du couplage antiferromagnétique $\mathrm{J}_{c}$.

\section{3 État 2 : état antiferro canté}

\subsubsection{Mesure des ondes de spin}

Lorsque l'on dope le composé pur par substitution de $\mathrm{La}$ par $\mathrm{Sr}$ ou $\mathrm{Ca}$, l'évolution de l'intensité des pics de Bragg magnétiques indique que la composante ferromagnétique le long de l'axe c croît, alors que la composante antiferromagnétique décroit. Lors des premières mesures de diffraction magnétique par Wollan et Koelher [1], l'existence d'un état canté proposé par de Gennes [6] (voir dessin Figure 4-c) a été remise en cause, et la possibilité d'une coexistence de phases ferromagnétiques et antiferromagnétiques a été suggérée. Si les mesures de diffraction ne peuvent répondre, la mesure des ondes de spin apporte une réponse plus précise.

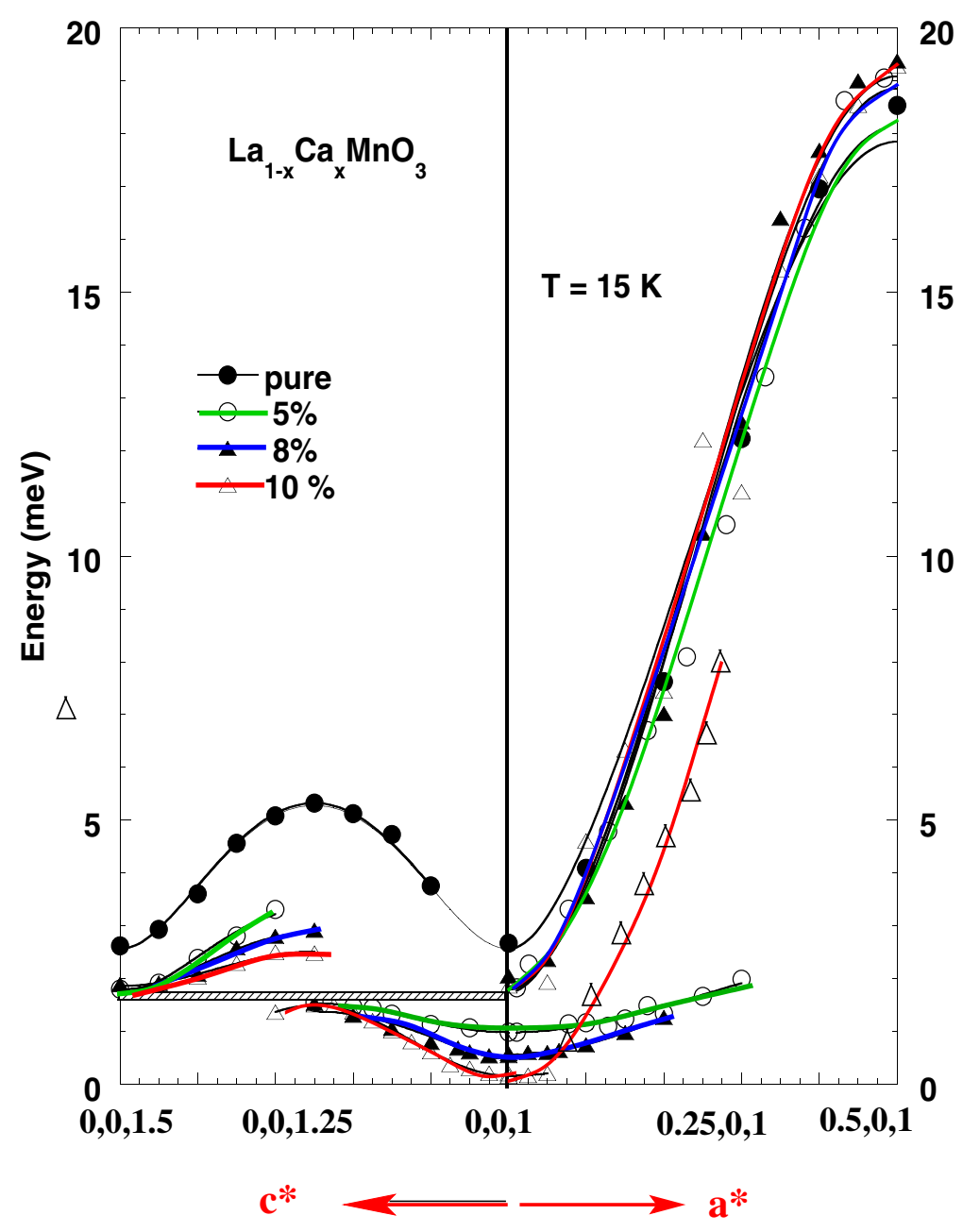

Figure 6. Comparaison des courbes de dispersion d'ondes de spin $\omega(\mathrm{q})$ des composés dopés au Ca avec $\mathrm{x}=0.05$, 0.08 et $\mathrm{x}=0.1$ et du composé pur. 

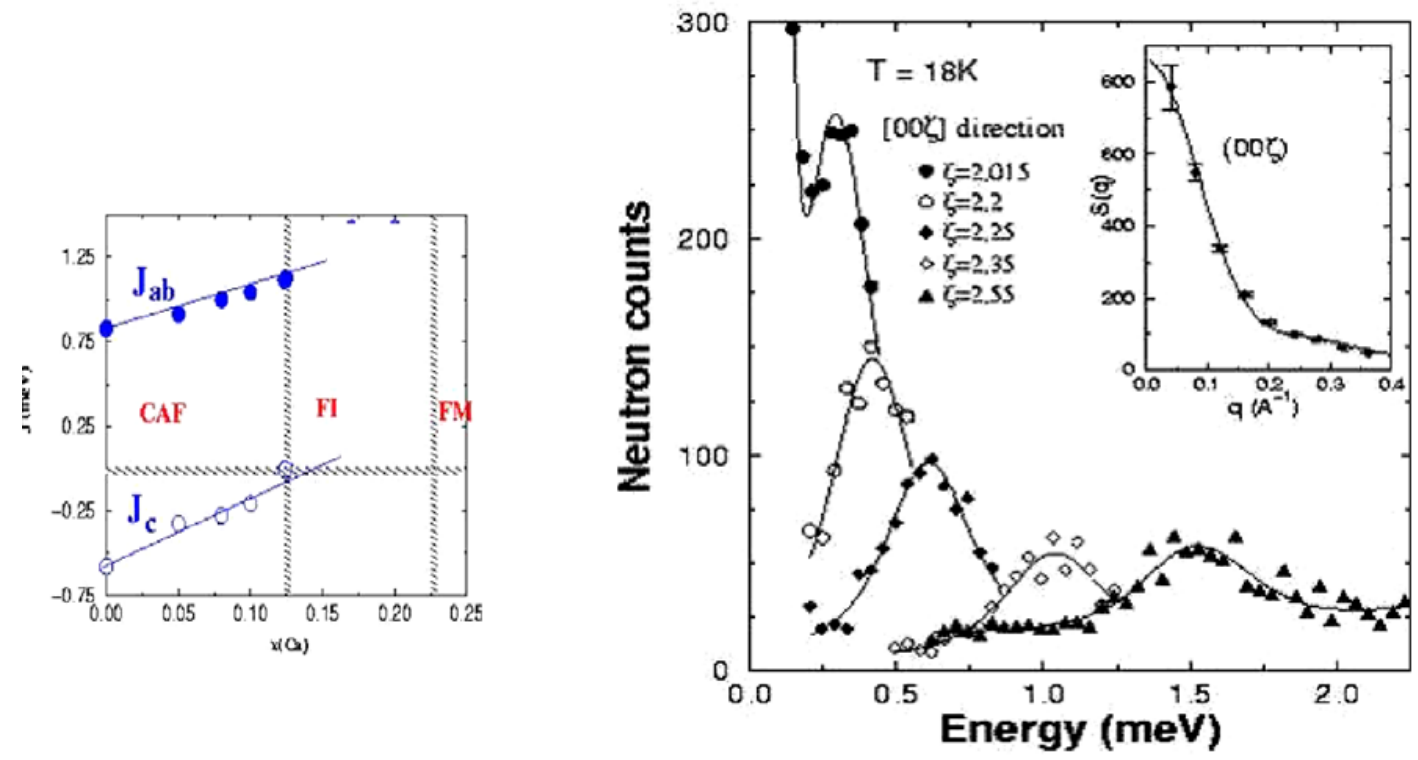

Figure 7. A gauche, évolution avec $x$ des couplages $\mathrm{J}_{a, b}$ et $\mathrm{J}_{c}$ de la branche à grand gap, déduite d'un Hamiltonien de Heisenberg. A droite: spectres bruts de la branche à petit gap selon $\mathbf{c}$.

Sur la figure 6 sont représentées les courbes de dispersion dans la direction $\mathbf{c}$ et la direction a ou $\mathbf{b}$, pour le composé pur (en noir), et pour les composés substitués au Calcium avec $\mathrm{x}=0.05,0.08$ et 0.1 (tracé vert, bleu, rouge) [8-10]. L'observation la plus frappante est l'apparition d'une nouvelle branche, au centre de zone, dans le gap d'une autre branche, similaire à celle du composé pur, avec cependant un gap à l'origine légèrement inférieur. Nous distinguons donc la branche à grand gap, qui rappelle le composé pur, et la branche -nouvelle- à petit gap.

La branche à petit gap indique l'apparition d'un couplage de nature ferromagnétique. Son intensité est en effet maximale au centre de zone ferromagnétique $\tau=(1,0,0)$, contrairement à la branche à grand gap qui, selon c, a une intensité maximale autour du $\tau_{A F}=(0,0,1.5)$. Sur la figure 7 , les spectres bruts correspondant à l'évolution des excitations selon [001] de cette branche à faible gap sont reportés pour $L a_{0.9} \mathrm{Ca}_{0.1} \mathrm{MnO}_{3}$, ainsi que la variation d'intensité intégrée $S(q)$ dans l'insert.

Lorsque la concentration en trous augmente:

- pour la courbe avec faible gap : le gap à $q=0$ décroit, et est presque nul à $\mathrm{x}=0.1$, le couplage ferromagnétique croît, comme l'indique la valeur croissante de la rigidité $\mathcal{D}$ dans l'approximation $\omega=\mathcal{D} q^{2}$ de la courbe de dispersion. Identique selon $\mathbf{c}$ et dans le plan pour $x=0.05,0.08$, il devient fortement anisotrope à l'approche de la transition métallique, $x=0.1$, c'est à dire beaucoup plus fort dans le plan (a, b) que selon $\mathbf{c}$.

- pour la courbe avec grand gap : le gap à $q=0$ reste constant, le couplage selon c de nature antiferromagnétique décroît en valeur absolue tandis que le couplage ferromagnétique le long de a ou b de cette même courbe augmente (voir l'évolution reproduite en utilisant le Hamiltonien de Heisenberg du composé pur, Fig 7-gauche). Considérant l'évolution des couplages pour les 2 courbes de dispersion, il apparait que la transition "phase cantée-phase ferromagnétique" a un caractère bidimensionnel pour les 2 types de branches.

On remarque que les 2 courbes de dispersion sont intimement liées le long de la direction c: l'intensité près du centre de zone ferromagnétique de la branche à grand gap, faible pour le composé pur, disparaît dans les composés dopés au bénéfice de l'autre branche. Par ailleurs, la valeur en énergie du grand gap 
(a)
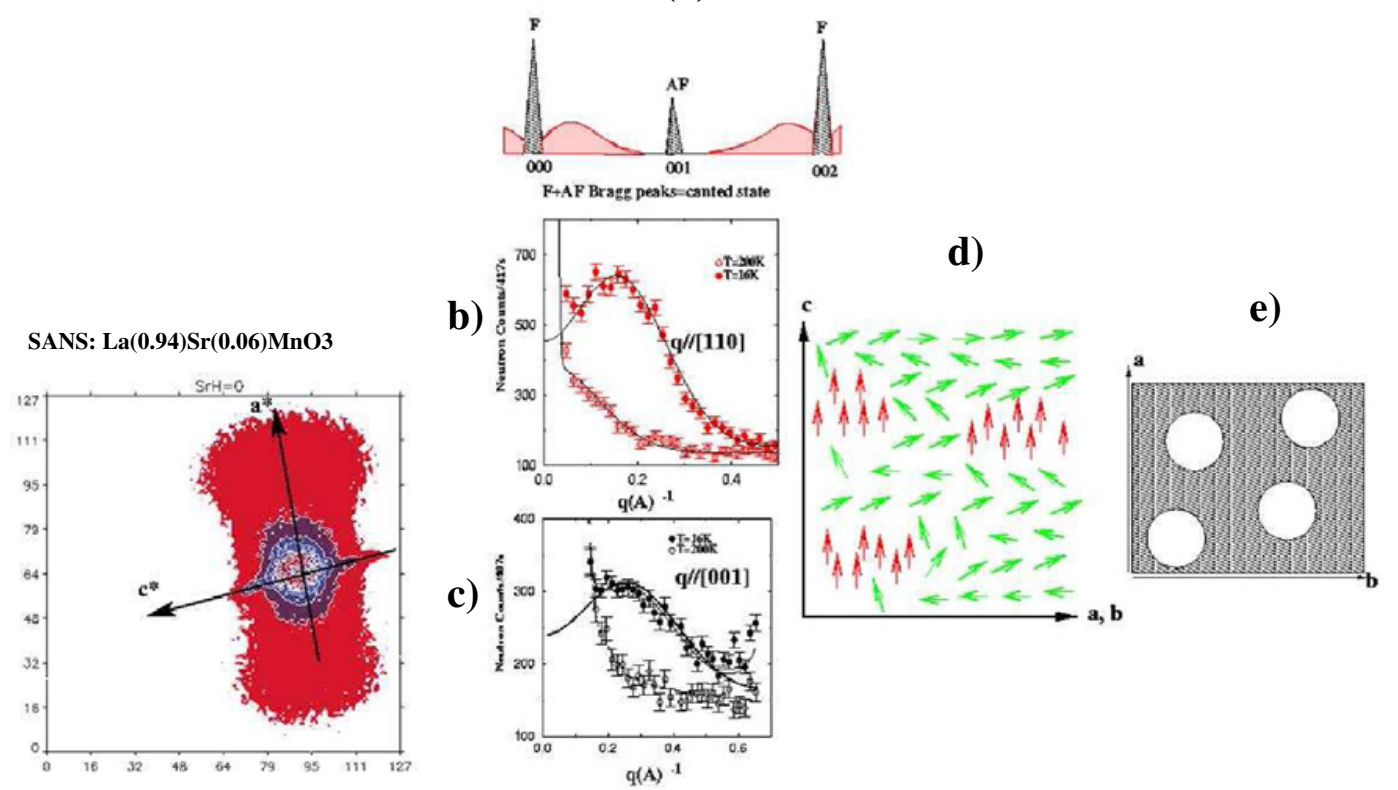

Figure 8. A gauche Diffusion aux petits angles du composé non mâclé $\mathrm{La}_{0.94} \mathrm{Sr}_{0.06} \mathrm{MnO} \mathrm{O}_{3}$ obtenue sur PAXY (LLB). A droite: a): schéma montrant la diffusion autour des pics de Bragg ferromagnétiques. b) et c): diffusion selon [100] et [001] respectivement autour du pic de Bragg (100), d): schéma de l'état magnétique canté modulé, e): schéma de la distribution de charge dans un plan $(\mathbf{a}, \mathbf{b})$.

reste contante avec $x$, de même que la valeur en énergie au point $q=0.25$ de la branche à faible gap. Cet effet se traduit par une courbure de la dispersion en ce point. avec l'existence d'un petit gap en énergie.

Il est clair que la courbe avec faible gap doit être associée au couplage ferromagnétique induit par les charges mobiles.

On pourrait comparer ce résultat avec les deux branches d'ondes de spin observées dans les isolants binaires antiferromagnétiques $M n_{(1-x)} C o_{x} F_{2}$ [11]. Dans ce cas, les 2 ions sont fixes dans le réseau. Dans le cas que nous considérons ici, les charges sont mobiles. Cela suggère fortement l'existence d'une dynamique spécifique des charges, soit une phase "métallique" inclue dans la matrice isolante, ou ségrégation de charge. C'est cette hypothèse que nous avons étudiée par diffusion aux petits angles.

\subsubsection{Diffusion aux petits angles et diffusion diffuse}

La figure 8-gauche représente la diffusion aux petits angles (PAXY, LLB) observée sur un composé $\mathrm{La}_{0.94} \mathrm{Sr}_{0.06} \mathrm{MnO}_{3}$ non mâclé à $T=14 \mathrm{~K}[12,13]$. La direction des axes cristallographiques est indiquée. On observe une diffusion dans la direction [100] ( $\left.\mathbf{a}^{*}\right)$ et non dans la direction de la composante ferromagnétique [001] (ou c*) de l'état canté. Cette extinction, liée à l'existence du facteur géométrique (seule les composantes magnétiques perpendiculaires au vecteur d'onde total peuvent être observées) indique que l'aimantation dans les inhomogénéités est orientée selon l'axe [001] ( $\left.\mathbf{c}^{*}\right)$ (direction du ferromagnétisme moyen induit par les charges).

La diffusion $I(q)$ observée autour de $\tau=(0,0,0)$ se retrouve autour de tous les pics de Bragg de nature ferromagnétique (cf Fig 8-a). Pour observer la diffusion non seulement le long de [100] mais aussi de [001], l'observation doit être faite, non pas autour $\tau=(0,0,0)$, mais dans une zone plus éloignée, 
telle que $\tau=(1,0,0)$ par exemple, soit $Q=q+\tau, \tau=(1,0,0)$, et $q$ selon [001]. Les figures 8-a,b montrent la diffusion observée autour de $\tau=(1,0,0)$, à la fois selon [100] (figure 8-b) (l'indication [110] dans la figure correspond à une indexation en maille orthorhombique [19]), et selon [001] (figure 8-c).

On remarque un maximum d'intensité à une valeur de $q_{0} \approx 0.18 \AA$, qui indique une distance caractéristique entre les inhomogénéités, selon a et $\mathbf{b}$, donc essentiellement dans les directions du plan (a, b). Par ailleurs la variation de $I(q)$, plus rapide selon [100] que selon [001] indique une extension de la diffusion plus grande dans le plan que selon c, c'est à dire, une anisotropie de forme. En utilisant le modèle de Guignier [12,13], on obtient $\xi \approx 17 \AA ̊$ selon [100] (soit $4 a$, où $a$ est le paramètre de maille), et $\xi \approx 7 \AA$, soit l'épaisseur d'un plan selon [001]. Nous concluons que les inhomogénéités ont un caractère bidimensionnel, probablement en liaison avec la règle de Hund, selon laquelle le saut des charges est facilité lorsque les spins ont la même orientation. Dans la structure en couches ferromagnétiques du composé parent, il est clair que les charges vont se déplacer plus facilement dans les plans (a, b) que selon c. Cette anisotropie de forme est à relier à l'anisotropie de couplage observée près de la transition (Figure 6).

La figure 8-c donne une représentation schématique que l'on peut se faire de ces inhomogénéités magnétiques : l'état canté est en fait un état canté modulé. La figure 8-d donne l'image correspondante de la répartition des charges avec des zones à caractère bidimensionnel métallique, dans une matrice isolante.

\subsubsection{Interprétation}

Grace à la diffusion aux petits angles, nous pouvons attribuer la branche basse à la dynamique de spins dans un modèle de charges itinérantes. Dans l'espace des phases pour lequel ce couplage est défini, la repartition spatiale inhomogène de la phase métallique n'intervient pas, car toutes les charges appartiennent à un réseau cohérent dans tout le cristal. Si la branche à petit gap correspond auc couplage magnétique défini par les charges mobiles dans le réseau réciproque, la branche à grand gap doit correspondre à une description du couplage magnétique dans l'espace direct, comme pour le composé pur (Hamiltonien de Heisenberg). Son évolution en fonction du dopage est bien reproduite dans le modèle de polarons de Feiner et Oles [14]. L'anomalie à $q=0.25$, qui sépare les 2 domaines en énergie (espaces direct et réciproque) révèle l'impossibilité de développer une onde progressive ferromagnétique pour $q \geq 0.25$, dans le modèle de charges itinérantes. Cette anomalie que nous retrouverons à toute les concentrations sera expliquée plus loin.

\section{4 État 3 : état ferromagnétique quasi-métallique $: x \approx 1 / 8$}

Pour $x>0.1$, c'est à dire dans la zone $x \approx 1 / 8$, pour les deux types de composés, la structure magnétique est devenue ferromagnétique (les pics de Bragg de nature antiferromagnétique ne sont plus observés). Un très grand changement apparaît pour les ondes de spins [15-18]. Deux régimes en température doivent être distingués, quasimétallique et isolant, associés à deux variations différentes de la résistivité.

Le domaine $T_{C}<T<T_{O O^{\prime}}$ où la résitivité indique un comportement "quasi-métallique" constitue l'état 3. Dans la figure 9 où sont reportés les spectres d'ondes de spin, on observe deux régimes en $q$ très similaires pour les deux composés: (i) $q \leq 0.25$ où apparaît une dispersion quadratique, caractéristique d'ondes propagatives de nature ferromagnétique et (ii) $q \geq 0.25$ où sont observés des niveaux peu dispersés, qui s'accrochent à des valeurs $\approx 7.5,12,17,23 \mathrm{meV}$ au bord de zone, pour les deux composés. Elles indiquent des excitations magnétiques plus localisées. En fait le plus bas niveau, à $\approx 7.5 \mathrm{meV}$ pourrait être le prolongement de la courbe dispersée, ce qui indiquerait essentiellement trois niveaux. Dans ce domaine en température, la courbe de dispersion définie près du centre $q=0$, s'extrapole à des énergies inférieures à celles des niveaux magnétiques. 


\section{Evolution avec $x$}
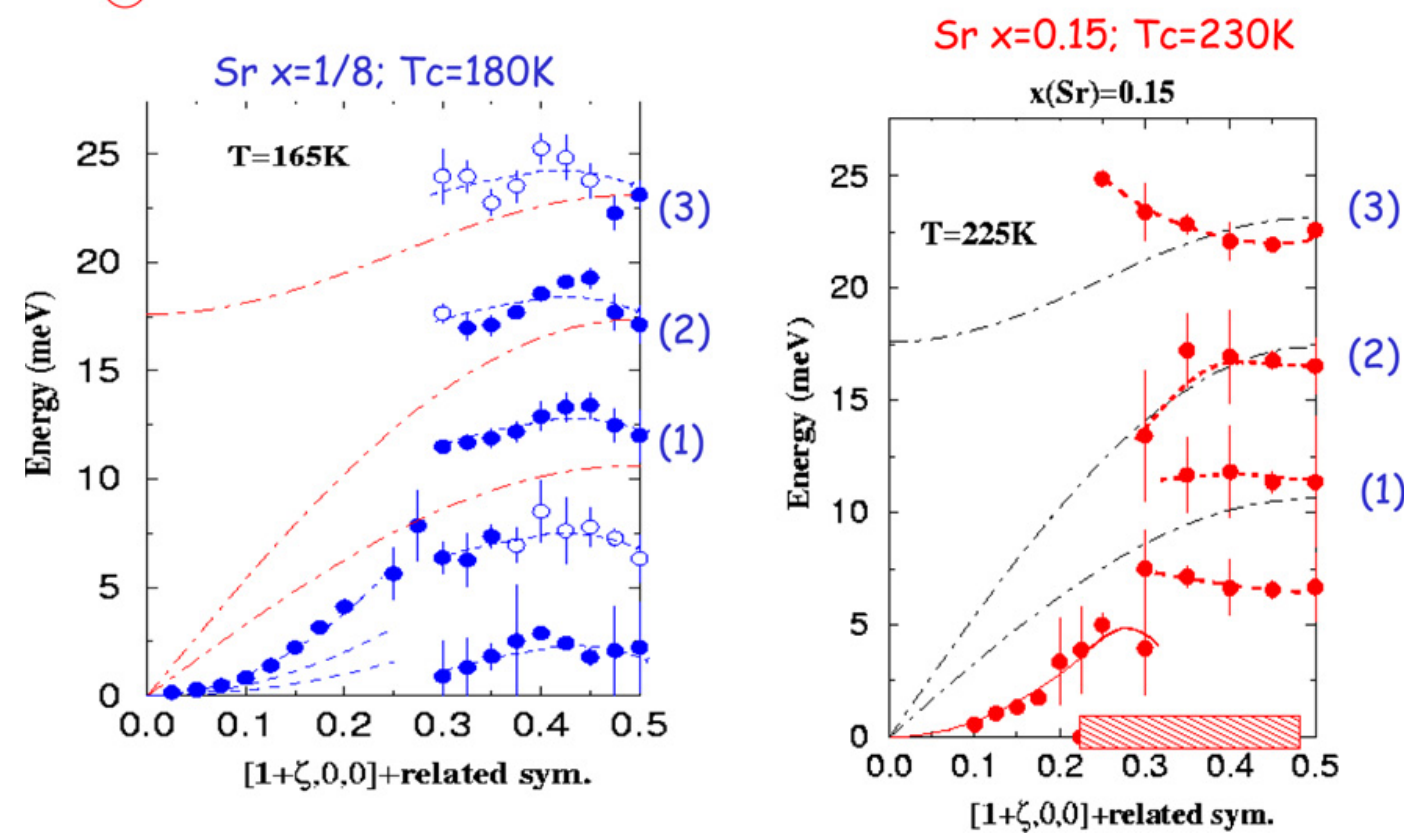

Figure 9. Etat quasi-métallique. A gauche Spectre d'ondes de spin de $L a_{0.875} \mathrm{Sr}_{0.125} \mathrm{MnO}_{3}$. A droite Spectre d'ondes de spin de $\mathrm{La}_{0.85} \mathrm{Sr}_{0.15} \mathrm{MnO}_{3}$.

Les courbes de dispersion de phonons (transverse acoustique TA, longitudinal acoustique LA et longitudinal optique LO), déterminées dans des zones de Brillouin éloignées sont indiquées par des courbes noires en pointillés. On observe une coincidence à $q=0.5$ avec les niveaux magnétiques pour LO, LA et TA.

La courbe de dispersion quadratique rappelle la dispersion à faible gap observée pour $x<0.125$. La constante de rigidité définie à partir de la courbure quadratique au centre de zone croit de manière régulière avec $x$ au travers de la transition isolant et quasi-métal du composé. Comme pour $x<0.125$, le comportement à petit q reflète les excitations magnétiques dans un modèle de charges itinérantes. Le changement de régime observé à $q>0.25$ révèle un cut-off pour ce modèle comme dans la phase précédente. Les niveaux observés caractérisent les défauts locaux, dans l'espace direct, dont l'influence apparait à $q>0.25$.

La figure 10 reproduit quelques spectres bruts dans ce régime de température intermédiaire. Pour $L a_{0.875} \mathrm{Sr}_{0.125} \mathrm{MnO}_{3}$, à $q=0.25$, on observe un mode large, tandis que pour $q=0.3$ et $q=0.35$ plusieurs modes apparaissent. Lorsque $q$ augmente, les positions des modes varient peu mais l'intensité se déplace des énergies plus faibles vers les énergies plus élevées.

Les excitations magnétiques ont été mesurées dans les autres directions de symétrie principales [110], [111]. Comme dans la direction [100], le spectre est constitué d'une courbe de dispersion quadratique aux petits $q$ et des niveaux à grand $q$ (cf Figure 12). Nous pouvons caractériser le caractère anisotrope des couplages dans les deux domaines en $q$. La figure 11 reproduit les courbes de dispersion observées à faible vecteur d'onde $q$ ( $q$ est exprimé en $\AA$ ) dans les 3 directions de symétrie pour le composé $\mathrm{La}_{0.875} \mathrm{Sr}_{0.125} \mathrm{MnO}_{3}$ (à gauche), et pour le composé $\mathrm{La}_{0.85} \mathrm{Sr}_{0.15} \mathrm{MnO}_{3}$ (à droite).

En fait le monocristal est mâclé, c'est à dire qu'il est constitué de multiples petits monocristaux pour lesquels les 3 axes du petit cube $\mathbf{a}, \mathbf{b}$, c sont superposés, alors que les couplages magnétiques dans les directions correspondantes, diffèrent. Les directions observables sont donc $[100]+[010]+[001]$, 
(3) $T_{0}, 0,1 T<T C$

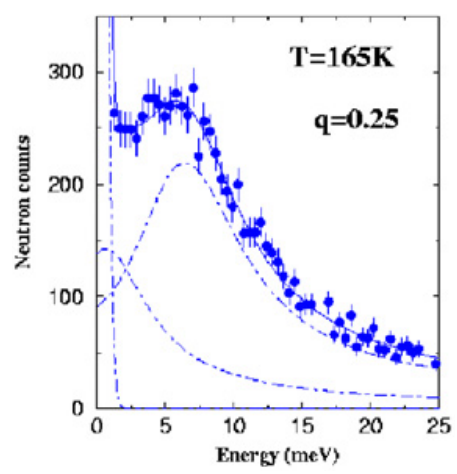

$X(S r)=0.125, T=165 K(T c=180 K)$

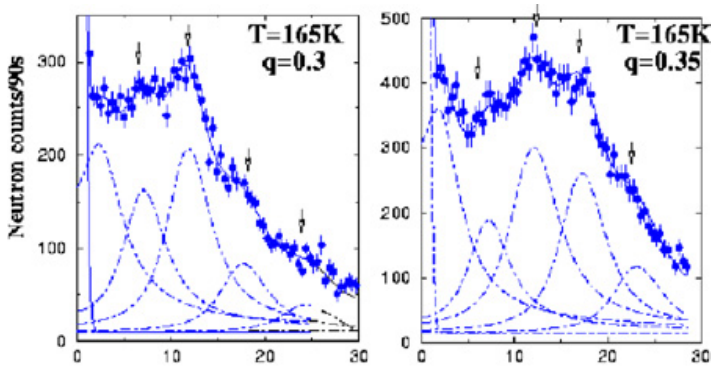

$X(S r)=0.15, T=225 K(T c=230 K)$

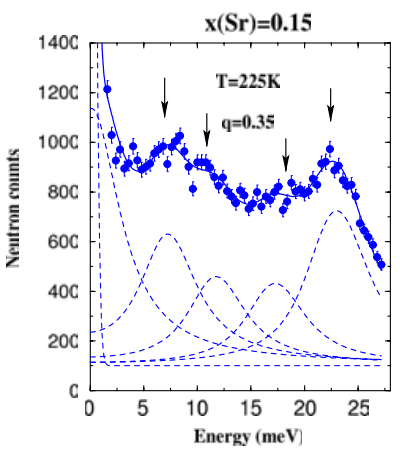

IN8, ILL

Figure 10. Etat 3, direction [100]. Spectres bruts, en haut: dans $L a_{0.875} \mathrm{Sr}_{0.125} \mathrm{MnO}_{3}$ à $q=0.25,0.3,0.35$. En bas: $L a_{0.85} \mathrm{Sr}_{0.15} \mathrm{MnO}_{3}$ à q $=0.35$.

(3) $x(S r)=0.125$

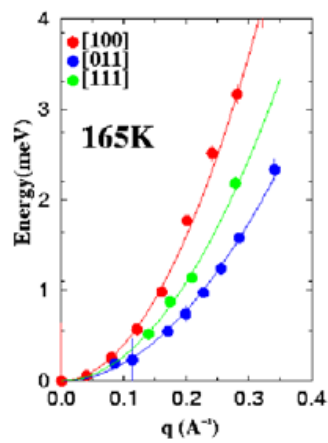

$x(S r)=0.125,=2 D$

\section{Régime petit $q \quad x(\mathrm{Sr})=0.15$}

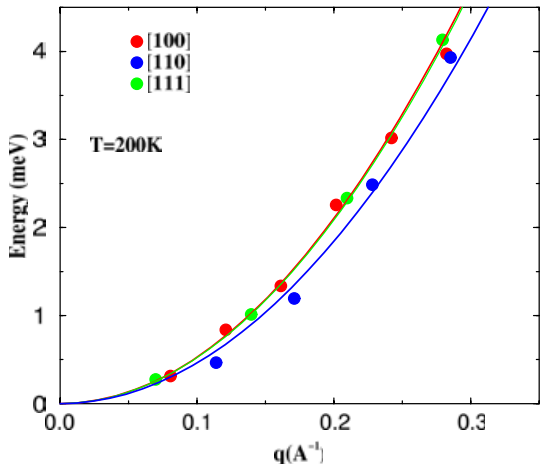

$x(S r)=0.15 \sim 3 D$

Figure 11. Etat 3. Comparaison des courbes de dispersion dans 3 directions de symmétrie à gauche pour $\mathrm{La}_{0.875} \mathrm{Sr}_{0.125} \mathrm{MnO}_{3}$, à droite pour $\mathrm{La}_{0.85} \mathrm{Sr}_{0.15} \mathrm{MnO}_{3}$. 


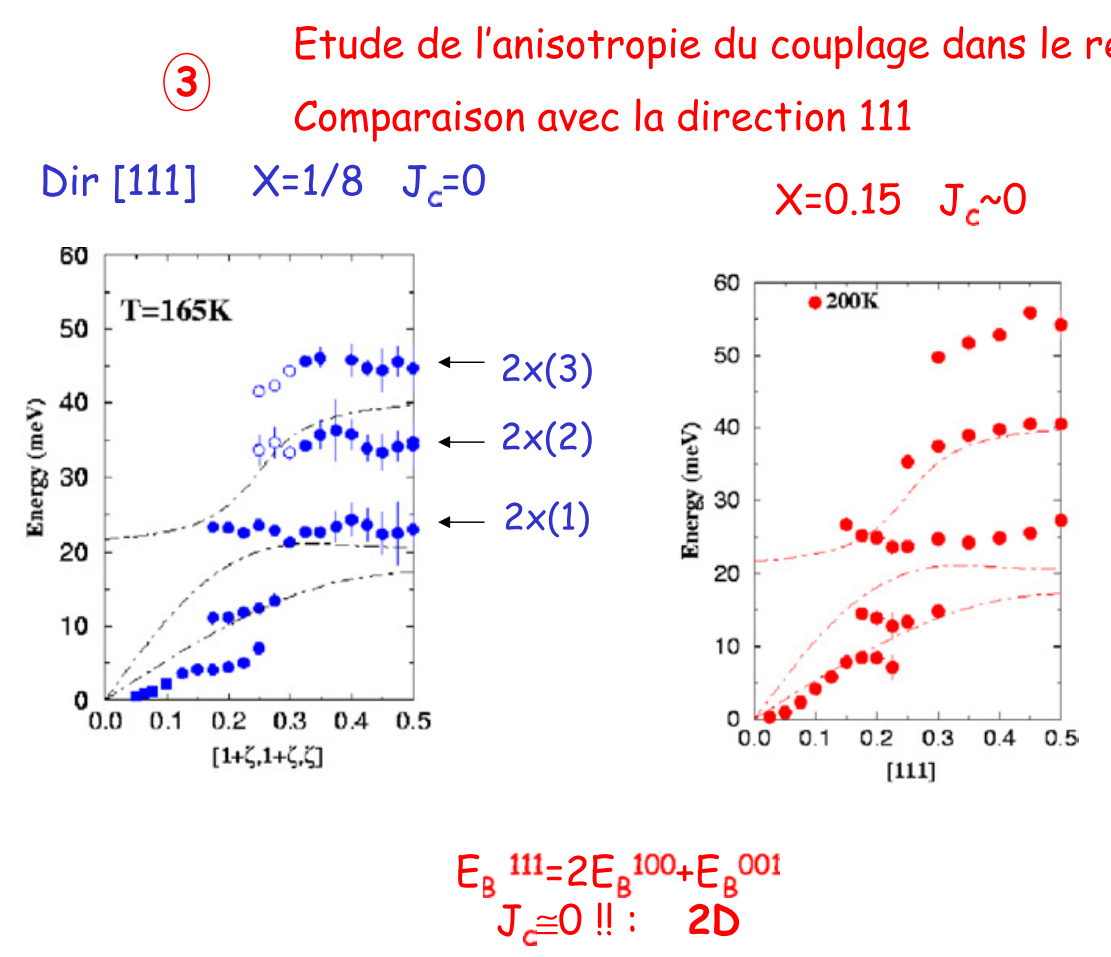

Figure 12. Etat 3. Spectre d'excitations magnétiques observé selon [111] pour $\mathrm{La}_{0.875} \mathrm{Sr}_{0.125} \mathrm{MnO}_{3}$ (à gauche) et $\mathrm{La}_{0.85} \mathrm{Sr}_{0.15} \mathrm{MnO}_{3}$ (à droite).

où seuls les domaines qui contribuent aux directions [100] et [010] sont équivalents pour le couplage magnétique, [101] $+[011]+[011]$ où seuls les domaines contribuant aux directions [101] et [011] sont également équivalents, et enfin, la direction [111] pour laquelle tous les domaines sont équivalents.

Dans le cas $x=0.125$, les valeurs en énergie des courbes de dispersion sont deux fois plus faibles selon [101] $+[011]+[011]$ que selon [100] $+[010]+[001]$, alors que la courbe selon [111] est intermédiaire. Ceci indique un caractère parfaitement bidimensionnel pour ce type de couplage (itinérant). En revanche, pour $x=0.15$, ce type de couplage apparait beaucoup plus isotrope.

La comparaison des énergies de bord de zone dans les différentes directions permet également de caractériser l'anisotropie du couplage associé aux niveaux $(q>0.25)$. Dans un Hamiltonien de Heisenberg, la valeur au bord de zone $q=0.5$ dans la direction [111] est la somme des valeurs observées à $q=0.5$ dans les trois directions du cube $([100]+[010]+[001])$. Nous savons que les contributions selon [100] et [010] sont identiques. Le facteur 2 trouvé entre ces 2 directions $(23 \mathrm{meV}$ et $46 \mathrm{meV}$ pour [100] et [111] respectivement) pour $\mathrm{La}_{0.875} \mathrm{Sr}_{0.125} \mathrm{MnO}_{3}$, permet de conclure à un caractère bidimensionnel. Cette observation est cohérente avec la variation du couplage magnétique avec $x$ selon c de l'état $x<0.125$, qui s'annule à la transition ferromagnétique (cf Figure 6, 7). Pour le cas de $\mathrm{La}_{0.85} \mathrm{Sr}_{0.15} \mathrm{MnO}_{3}$ les 2 valeurs (23 meV et $54 \mathrm{meV}$ ) indiquent un facteur différent de 2, mais cependant loin de l'isotropie.

\section{5 État 4 : état ferromagnétique isolant, $x \approx 1 / 8$}

\subsubsection{Expérience}

Le domaine $0<T<T_{O O^{\prime}}$ correspond au domaine de température où la résistivité présente un comportement isolant. Les figures 13 et 14 montrent la dispersion des ondes de spin selon [100] pour 
(4) $\mathrm{La}_{7 / 8} \mathrm{Sr}_{1 / 8} \mathrm{MnO}_{3}$

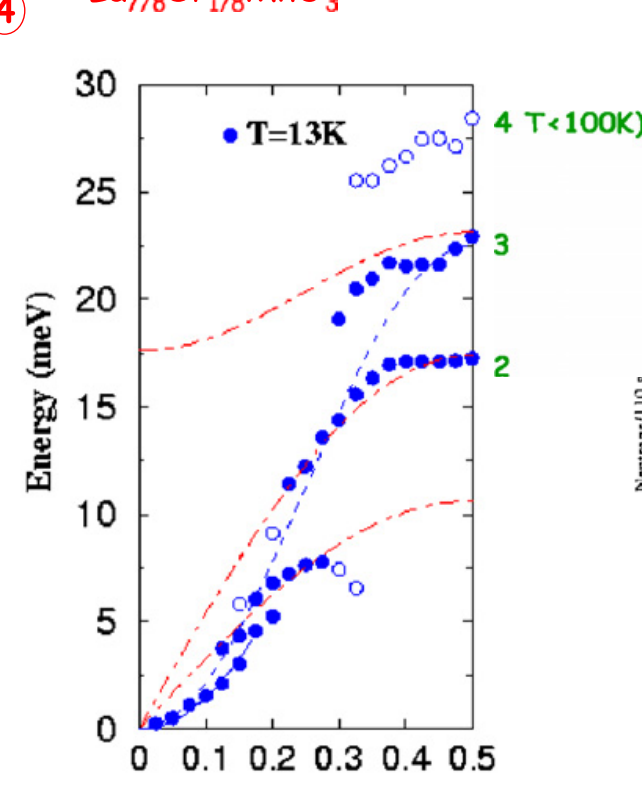

$[0,0,1+\zeta]+$ related symmetry

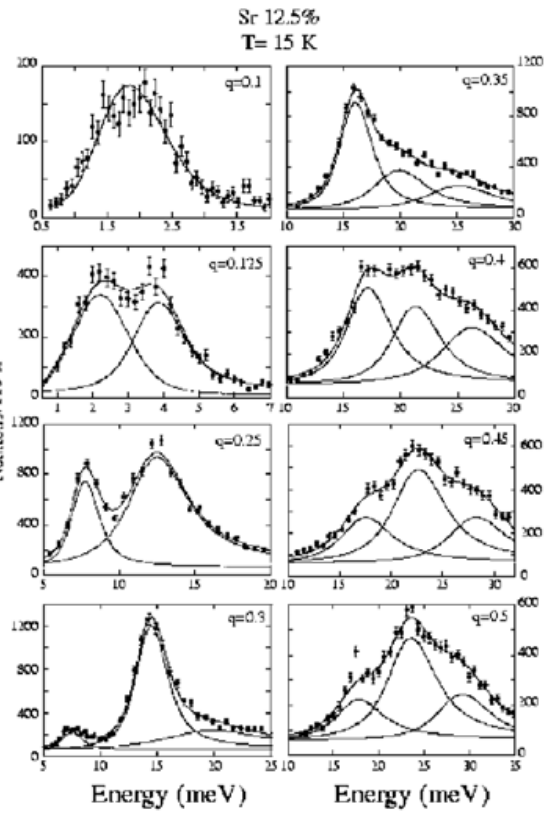

Figure 13. Etat 4, direction [100]. A gauche: Spectre d'ondes de spin mesuré dans $L a_{0.875} S r_{0.125} M n O_{3}$ A droite : spectres bruts correspondants, pour différentes valeurs de q.

(4)
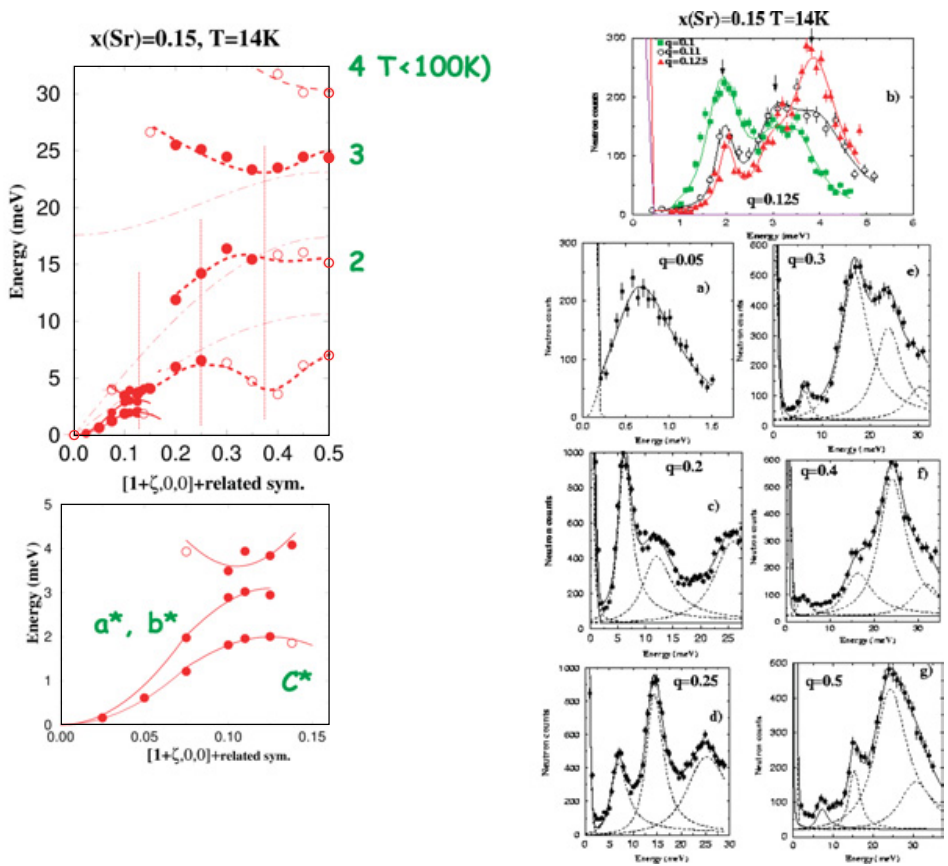

Figure 14. Etat 4, direction [100].A gauche: Spectre d'ondes de spin mesuré dans la direction [100] pour $\mathrm{La}_{0.85} \mathrm{Sr}_{0.15} \mathrm{MnO}_{3}$. A droite : spectres bruts correspondants, pour differentes valeurs de q. 
$\mathrm{La}_{0.875} \mathrm{Sr}_{0.125} \mathrm{MnO}_{3}$ et $\mathrm{La}_{0.85} \mathrm{Sr}_{0.15} \mathrm{MnO}_{3}$, avec les spectres bruts correspondants [20,21]. Pour les 2 composés, l'état isolant se caractérise ainsi :

Dans la direction $[\mathbf{1 0 0}]+[\mathbf{0 1 0}]+[\mathbf{0 0 1}]$ : un petit gap s'ouvre autour de $q=0.125$, indiquant une nouvelle périodicité de $4 a$ pour le couplage de la région quadratique. Il est important de déterminer si ce gap concerne les 3 directions de symmétrie. Cette information est malheureusement très difficile à obtenir dans le cas d'un composé maclé. Les mesures dans $\mathrm{La}_{0.875} \mathrm{Sr}_{0.125} \mathrm{MnO}_{3}$ ne sont pas incompatibles avec cette conclusion, mais sans certitude. Par contre, dans le cas de $\mathrm{La}_{0.85} \mathrm{Sr}_{0.15} \mathrm{MnO}_{3}$, où 3 modes ont pu être séparés autour de $q=0.125$ ( $\mathrm{cf}$ figure 14-gauche pour la dispersion et figure 14-droite pour les spectres bruts), la situation expérimentale apparait plus claire. La courbe d'énergie inférieure qui disperse faiblement et disparait à $q>0.15$ correspond à la direction $\mathbf{c}$ ou [001], ce qui signifie que le gap à $q=0.125$ concerne seulement les directions a et $\mathbf{b}$ du plan. Au-delà de cette valeur de $q$, trois niveaux modulés en $q$ sont observés. On eut également distinguer, dans les 2 composés, une autre excitation à une énergie supérieure, située à $\mathrm{E} \approx 31 \mathrm{meV}$, de très faible en intensité.

Ces niveaux sont plus dispersés dans le cas de $\mathrm{La}_{0.875} \mathrm{Sr}_{0.125} \mathrm{MnO}_{3}$, avec une coincidence frappante avec les courbes de dispersion acoustiques et optique des branches de phonons TA, LA et LO. Dans $\mathrm{La}_{0.85} \mathrm{Sr}_{0.15} \mathrm{MnO}_{3}$, où cette coincidence est un peu moins nette, on observe des modulations qui suggèrent une périodicité de $4 a$. Celles-ci ont été matérialisée par des lignes verticales dans la figure 14gauche.

Enfin, si l'on trace la courbe de dispersion de type "cosinus" que l'on attendrait pour un ferromagnétique classique, en extrapolant la courbe de dispersion observée au centre de zone, on observe une "adéquation" parfaite entre la partie centre de zone (description avec charges itinérantes) et les niveaux (sauf celui faible à $31 \mathrm{meV}$ ).

Dans la direction [111] étudiée avec une bonne résolution $(\Delta E=3 \mathrm{meV})$ pour $\mathrm{La}_{0.85} \mathrm{Sr}_{0.15} \mathrm{MnO}_{3}$, on peut distinguer 6 niveaux distincts (cf figure 15). La valeur en énergie du bord de zone, $E=55 \mathrm{meV}$, très proche du double de celle observée dans la direction (100] (figure 14-gauche), suggère la persistance d'un couplage bidimensionnel dans l'état isolant $\mathbf{4}$ basse température.

\subsubsection{Interprétation}

L'interprétation concerne le composé $L a_{0.85} \mathrm{Sr}_{0.15} \mathrm{MnO}_{3}$, pour lequel les caractéristiques expérimentales sont établies avec certitude. Deux origines peuvent être données pour l'observation des niveaux: ondes confinées dans un amas de taille finie isolé, ou fluctuations quantiques d'un spin global associant plusieurs spins fortement couplés.

Dans le cas présent l'ouverture d'un gap à $q=1 / 8$, suggère l'existence de petits amas ferromagnétiques de taille $\xi=4 a$ pour lesquels on s'attend à des ondes confinées. En l'absence d'indication expérimentale, on ne considère pas d'organisation selon c (couplage supposé bidimensionnel), et l'on considère un couplage identique selon $\mathbf{a}$ et $\mathbf{b}$, comme nous l'avons observé à toute concentration. Dans la figure 16, les points rouges représentent les atomes de $\mathrm{Mn}$, les points bleus ou verts, les atomes d'oxygène, $\mathrm{O}$, qui les entourent. L'expérience de neutrons ne donne des informations que sur la dynamique des spins et non sur les positions des charges. Comme l'origine des amas est liée à la valeur de la concentration $x \approx 1 / 8$, nous obtenons 4 charges par domaine. Dans le dessin de la figure 16 , à gauche, ceux-ci sont représentés à la frontière des domaines, comme dans le modèle proposé pour les supraconducteurs à haut $\mathrm{T}_{C}$ [19]. On peut aussi imaginer que les 4 charges sont à l'intérieur du domaine, fortement corrélées, induisant par leur mobilité un fort couplage à l'intérieur du domaine, et faible entre les domaines.

Le calcul des excitations dans un petit domaine de taille $4 a \times 4 a$ a été effectué avec un Hamiltonien de Heisenberg, dans lequel on définit 2 couplages entre premiers voisins: $\mathrm{J}_{O O}$ qui couple les Mn à l'intérieur des amas et $\mathrm{J}_{\text {inter }}$ qui couple les $\mathrm{Mn}$ d'un amas à l'autre. La forme analytique des niveaux 


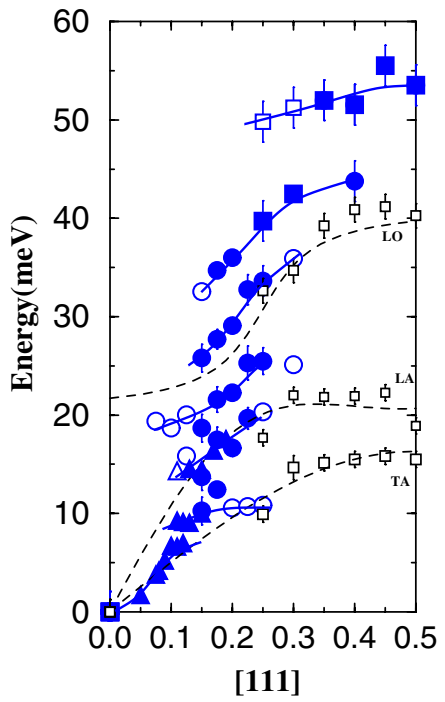

Figure 15. Etat 4. Spectre d'ondes de spin mesuré dans la direction [111] pour $L a_{0.85} \mathrm{Sr}_{0.15} \mathrm{MnO}_{3}$.
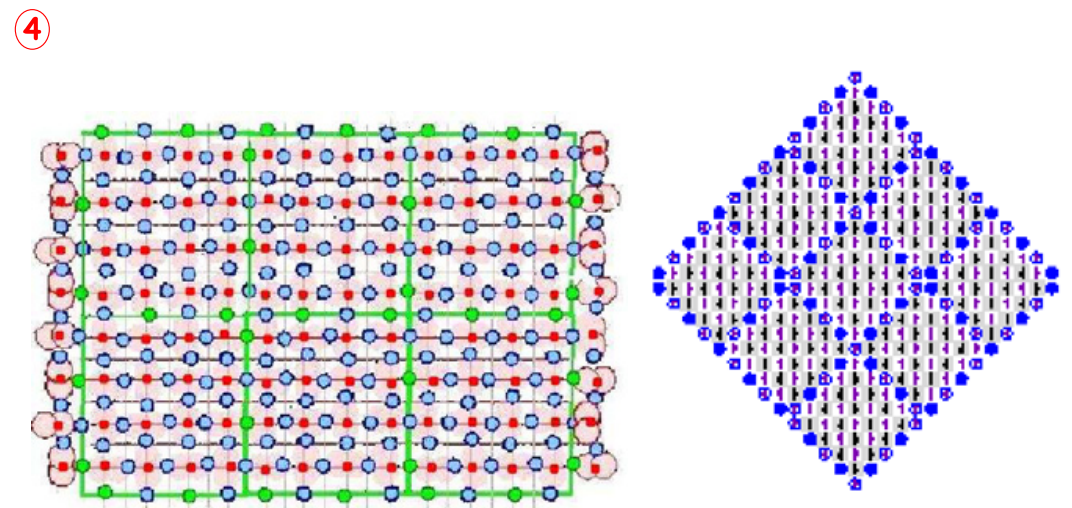

Modèle proposé

pour supra

(Fine, 2004)

Figure 16. Etat 4. A gauche, représentation schématique du modèle d'amas ordonnés. A droite: représentation schématique du modèle d'ordre de charges ou "stripes" proposé pour les supraconducteurs à haute température.

s'écrit [18] :

$$
E_{x, y}=4 J_{O O} S\left(1-\frac{\cos \frac{\pi x}{n}+\cos \frac{\pi y}{n}}{2}\right)
$$

avec $n=4$ and $x, y=0, \ldots 3$. Ce modèle permet de retrouver 3 niveaux selon [100] et 6 niveaux selon [111]. On obtient un accord presque quantitatif avec $\mathrm{J}_{O O}=1,7 \mathrm{meV}$ et $\mathrm{J}_{\text {inter }}=\mathrm{J}_{O O} / 5$, comme le montre la figure 17. Ce modèle, avec des constantes de couplage "effectives" ne permet cependant pas de comprendre la coincidence entre les niveaux magnétiques et les branches de phonons dispersées, cette coincidence étant associée à la physique du système de spins et de charges. 

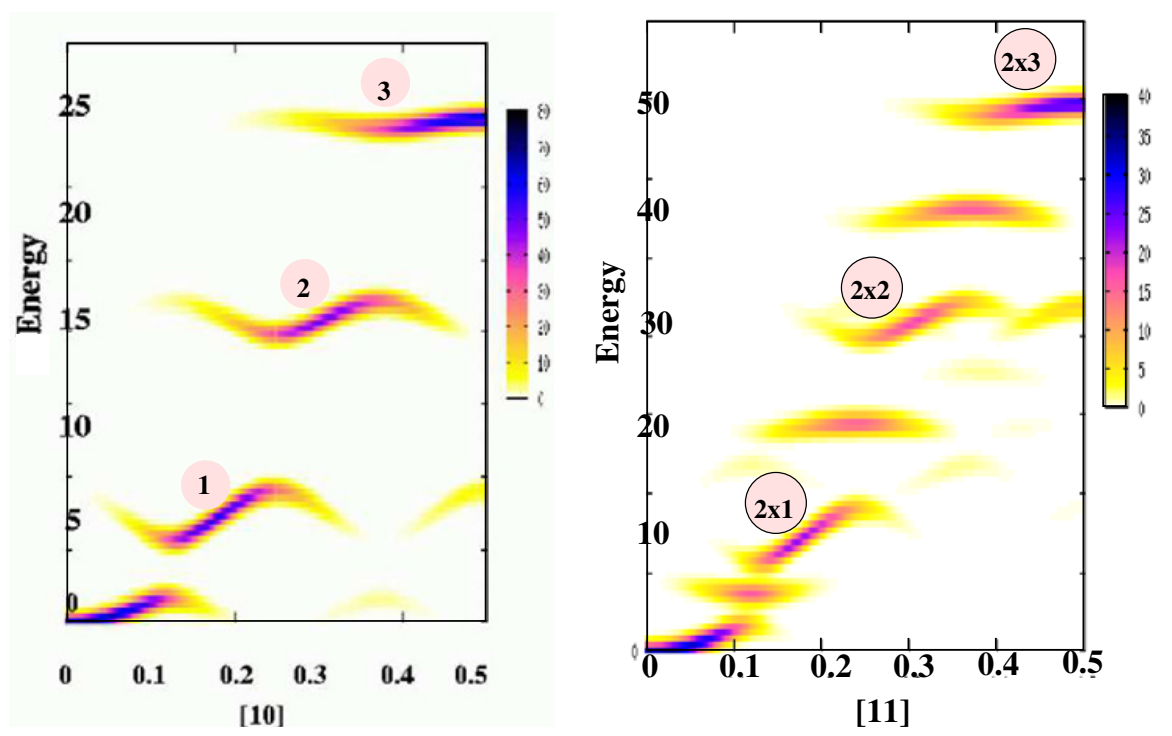

Figure 17. Courbe d'ondes de spin calculée dans le modèle de la figure 16, à gauche selon [100] et à droite selon [111].

\section{6 État 5 : état ferromagnétique métallique}

\subsubsection{Expérience}

Au-delà de la phase $x \approx 1 / 8$, les mesures de résistivité indiquent un état véritablement métallique. Il existe un grand nombre de mesures des excitations magnétiques dans cet état. Tous indiquent que les composés métalliques ont des courbes d'ondes de spin très anormales, spécialement au bord de zone (voir figure 18) [21-23]. Alors que pour un modèle de bande [20] on s'attend à une courbe unique de type "fonction cosinus", la courbe de dispersion présente au bord de zone un amollissement ("softening"), associé à un élargissement des modes. Beaucoup d'interprétations ont été proposées: couplage magnonphonon, changement d'ordre orbital, effet d'un couplage à plus grande distance etc. [26-28].

Nous avons entrepris d'examiner les ondes de spins avec une haute résolution $(\Delta E=3 \mathrm{meV})$ en utilisant des monochromateurs de $\mathrm{Cu}\left(\mathrm{Cu}_{200,111,220}\right)$. Dans la figure 19 sont reportées, à droite, les dispersions d'ondes de spin des composés métalliques $\mathrm{La}_{1-x} \mathrm{Sr}_{x} \mathrm{MnO}_{3}(x(\mathrm{Sr})=0.2, x(\mathrm{Sr})=0.3)$ et $\mathrm{La}_{0.7} \mathrm{Ca}_{0.3} \mathrm{MnO}_{3}$, et à gauche, les spectres brut correspondants, pour $q=0.5$ [23]. Comme nous l'avons observé dans les précédents états ferromagnétiques, la courbe de dispersion est constituée d'une partie quadratique au centre de zone caractéristique d'un milieu ferromagnétique tridimensionnel, et, au bord de zone, de plusieurs niveaux plus ou moins dispersés en $q$. Les deux niveaux à faible énergie se situent aux valeurs $E \approx 15,22 \mathrm{meV}$, comme dans les états magnétiques décrits précédemment (états 3 et 4 ), correspondant aux valeurs de LA et LO à $q=0.5$. Par ailleurs, de nouveaux niveaux apparaissent à des valeurs d'énergie supérieures, $E \approx 32,41 \mathrm{meV}$, ce qui indique essentiellement quatre niveaux (la valeur $51 \mathrm{meV}$ observée pour $\mathrm{x}(\mathrm{Sr})=0.3$ seulement, n'a pas été confirmée, et pourrait etre liée à une fuite du faisceau direct). Si l'on suit l'intensité principale de la courbe de dispersion, on apercoit un changement de régime autour de $q=0.25$ faible pour $x(S r)=0.2$ devenant très prononcé lorsque la concentration en trous augmente $(x(S r)=0.3)$. Contrairement aux deux situations décrites précédemment, la courbe définie au centre de zone s'extrapole à des valeurs situées au-dessus des niveaux principaux. Le niveau à $41 \mathrm{meV}$ pourrait être la trace de cette extrapolation au bord de zone. 


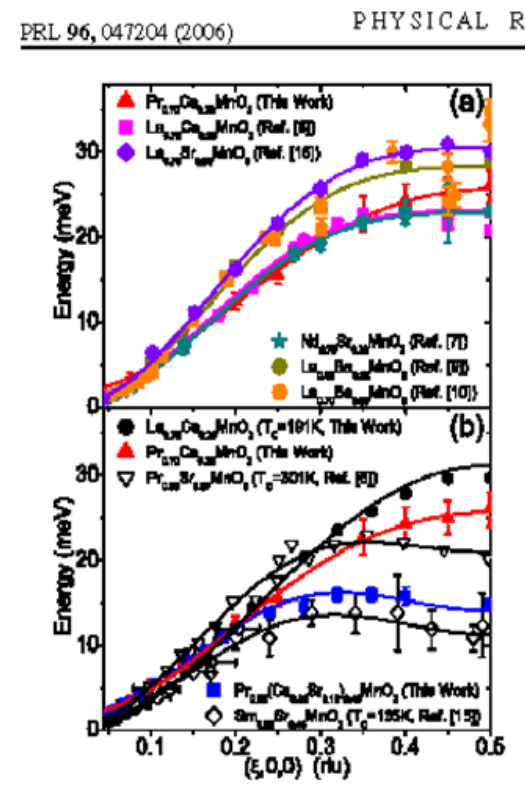

Figure 18. Courbes de dispersion montrant l'effet d'amollissement au bord de zone observé dans un grand nombre de composés métalliques (voir Ye et al. [27]).

Dans la direction [111] de nouveaux niveaux sont également observés jusqu'à $\mathrm{E} \approx 100 \mathrm{meV}$, indiquant un couplage de nature $3 D$.

\subsubsection{Interprétation}

Un tel changement de régime autour de $q=0.25$ ne peut être expliqué par un modèle de Heisenberg avec des couplages à longue distance comme cela a été récemment proposé. De même le modèle d'amas de taille $4 a$ dispersés au sein de la matrice de spins métallique, que nous avions suggéré en considérant les similarités des niveaux dans les différentes phases [18], ne peut pas être généralisé dans un grand domaine de concentration. De toute évidence, les défauts magnétiques à l'origine des anomalies d'ondes de spin à $q=0.25$ observée dans les 4 domaines décrits ci-dessus, doivent être liés à la physique fondamentale de ces systèmes, comme nous le décrivons de manière qualitative ici. Le couplage de double-échange introduit par Zener [5], indique que la charge qui se déplace selon un des axes du petit cube, couple très fortement une paire de spins, formant un "lien" fort, de spin total $S=7 / 2$, qui possède des excitations observables $(\Delta S=1)$. La mobilité de ce lien implique l'absence de lien premier voisin, de sorte que le défaut magnétique mobile associé au saut de la charge comprend 3 sites proches voisins du réseau $(\mathrm{i}, \mathrm{j}, \mathrm{k})$, avec une paire de spins en $(\mathrm{i}, \mathrm{j})$ fortement couplés, et une paire en $(\mathrm{j}, \mathrm{k})$ non couplée. Cette excitation ou "défaut dynamique", de taille $2 a$ perturbe la courbe d'onde de spin de type métallique pour $q>0.25$. La coincidence entre les niveaux magnétiques et phonons à $\mathrm{q}=0.5$, implique l'assistance de phonons acoustiques et optique ( TA,LA,LO) à $q=0.5$ dans le saut de charge, (ou le déplacement du lien). Trois régimes sont observés. Dans le cas d'une très faible mobilité (région quasi-élastique), les énergies des phonons sont au-dessus de l'onde de spin de type métallique. L'effet à $q=0.25$ est important. Par contre, dans le cas d'une forte mobilité (état métallique), l'onde de spin se développe au-travers et au-dessus des énergies de phonons, de sorte que l'anomalie à $q=0.25$ est faible pour les faibles dopages $(\mathrm{x}(\mathrm{Sr})=0.2)$, devenant forte lorsque le 

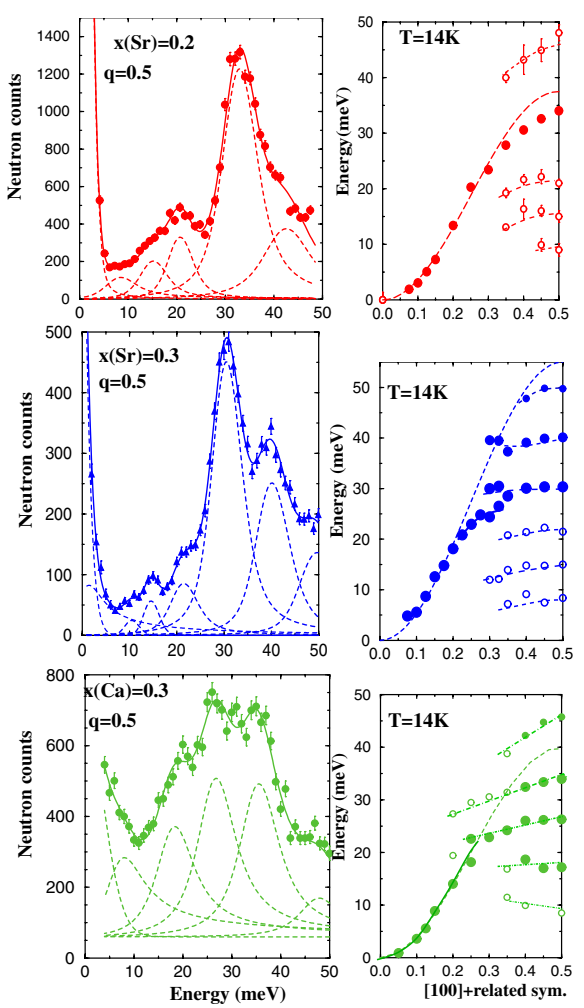

Figure 19. A droite, courbes de dispersion d'ondes de spin observée dans $L a_{1-x} S r_{x} M n O_{3}(x(S r)=0.2, x(S r)=$ 0.3 ) et $\mathrm{La}_{0.7} \mathrm{Ca}_{0.3} \mathrm{MnO}_{3}$. A gauche, spectre brut observé à q $=0.5$ correspondant.

nombre de charges augmente $(\mathrm{x}(\mathrm{Sr})=0.3)$. Enfin, dans le cas où les deux domaines d'énergie peuvent s'accorder, c'est à dire autour de $x=1 / 8$ à la transition $T_{o o^{\prime}}$, la coincidence des ondes propagatives de phonons et de magnons observée pour $q \geq 1 / 8$, suggère un déplacement des paires de spin de Zener avec les ondes élastiques propagatives, ce qui correspond à une forte corrélation de charges sur une échelle de taille $4 a$. L'anomalie à $q=0.25$ dans la phase cantée isolante, résulte du même effet: défaut magnétique associé aux paires de spin mobiles dans les “zones métalliques”, qui dans l'espace des phases sont toutes connectées par la cohérence du réseau.

\section{CONCLUSION}

En conclusion, l'évolution des ondes de spin en fonction du dopage permet d'avoir une image simple et unifiée de l'ensemble des observations. A toute concentration on distingue deux types de dynamique l'une associée au modèle de charges itinérantes $(q \leq 0.25)$, l'autre associée à des défauts dans l'espace direct $(q \geq 0.25)$ que nous pouvons comprendre par l'effet du couplage de Zener où la mobilité est assistée par des phonons.

\section{Remerciements}

Ce travail a été fait en étroite collaboration avec F. Moussa et S. Petit du laboratoire Léon Brillouin. Il a fait l'objet de deux thèses, G. Biotteau et P. Kober-Lehouelleur. Les monocristaux ont été obtenus grâce à L. Pinsard, de l' université d'Orsay et Y. Mukovskii de l'institut MISIS (Moscou, Russie). 


\section{Références}

[1] E. O. Wollan et W. C. Koehler Phys. Rev., 100, 545 (1955)

[2] A. Urushibara et al. Phys. Rev. 51, 14103 (1995)

[3] P. Schiffer et al. Phys. Rev. Lett. 753336 (1995)

[4] Nanoscale Phase Separation and Colossal Magnetoresistance, edited by E. Dagotto (SpringerVerlag, Berlin, 2002)

[5] V. Kiryukhin et al. Phys. Rev. B 70, 214424 (2004)

[6] C. Zener Phys. Rev. 81, 440 (1951), Phys. Rev. 82, 403 (1951)

[7] P.-G. de Gennes Phys. Rev. 118, 141 (1960)

[8] F. Moussa M. Hennion J. Rodriguez-Carvajal, H. Moudden L. Pinsard and A. Revcolevschi, Phys. Rev. B 5415149 (1996)

[9] M. Hennion, F. Moussa, J. Rodriguez-Carvajal, L. Pinsard and A. Revcolevschi Phys. Rev B 56 R497 (1997)

[10] F. Moussa, M. Hennion G. Biotteau J. Rodriguez-Carvajal, L. Pinsard and A. Revcolevschi Phys. Rev. B 6012299 (1999)

[11] G. Biotteau, M. Hennion, F. Moussa, J. Rodriguez-Carvajal, L. Pinsard, A. Revcolevschi Y. M. Mukovskii and D. Shulyatev, Phys. Rev. B 64104421 (2001)

[12] R. A. Cowley AIP Proceedings, 29 (1975)

[13] M. Hennion F. Moussa G. Biotteau J. Rodriguez-Carvajal L. Pinsard A. Revcolevschi Y. M. Mukovskii and D. Shulyatev Phys. Rev Lett. 811957 (1998)

[14] M. Hennion F. Moussa G. Biotteau J. Rodriguez-Carvajal L. Pinsard and A. Revcolevschi Phys. Rev. B 61, 9513 (2000)

[15] L. F. Feiner and A. Oles Physica B 259-261, 796

[16] M. Hennion et al. Phys. Rev. Lett. 94057006 (2005)

[17] M. Hennion F. Moussa P. Lehouelleur P. Reutler and A. Revcolevschi Phys. Rev. B 73, 104453 (2006)

[18] F. Moussa M. Hennion F. Wang P. Kober J. Rodriguez-Carvajal, P. Reutler L. Pinsard and A. Revcolevschi Phys. Rev. B 67214430 (2003)

[19] S. Petit et al. Phys. Rev. Lett 102207201 (2009)

[20] Boris Fine Cond-mat/0404488

[21] N. Furukawa J. Phys. Soc. Jpn 651174 (1996)

[22] Y. Endoh et al. Phys. Rev. Lett. 94, 017206 (2005)

[23] F. Ye et al. Phys. Rev. Lett. 96, 047204 (2006) and Phys. Rev. B 75144408 (2007)

[24] F. Moussa et al. Phys. Rev. B 76064403 (2007) 